\title{
Antagonizing the metabolic hormone cholecystokinin (CCK) and its receptor CCKAR in the lung abolishes obesity-induced airway hyperresponsiveness
}

\section{Ronald Pangniban}

Harvard University

\section{Maoyun Sun}

Harvard T.H. Chan School of Public Health

Chan Young Park

Harvard School of Public Health

\section{Alvin Koh}

Channing Division of Network Medicine, Department of Medicine, Brigham and Women's Hospital

\section{David Kasahara}

Harvard School of Public Health

\section{Elliot Israel}

Brigham and Women's Hospital, Harvard Medical School

\section{Marc Hershenson}

University of Michigan

\section{Scott Weiss}

Brigham and Women's Hospital, Harvard Medical School,

\section{Blanca Himes}

University of Pennsylvania

\section{Jeffrey Fredberg}

Department of Environmental Health, Harvard T.H. Chan School of Public Health, Boston, MA, USA https://orcid.org/0000-0002-7968-0920

\section{Kelan Tantisira}

UCSD

\section{Stephanie Shore}

Harvard T.H. Chan School of Public Health

Quan Lu ( $\nabla$ qlu@hsph.harvard.edu )

Harvard University 
Keywords: obesity-asthma, cholecystokinin (CCK), CCKAR, airway hyperresponsiveness (AHR), RNA-seq transcriptomic data

Posted Date: September 8th, 2021

DOI: https://doi.org/10.21203/rs.3.rs-841619/v1

License: (c) (1) This work is licensed under a Creative Commons Attribution 4.0 International License. Read Full License

Version of Record: A version of this preprint was published at Nature Communications on January 4th, 2023. See the published version at https://doi.org/10.1038/s41467-022-35739-8. 


\section{Abstract}

Obesity is a significant co-morbidity that associates with increased prevalence and severity of asthma. The mechanism underlying the obesity-asthma association remains poorly understood, and for obese asthmatics there is no effective therapy for obese asthmatics. Here we show that cholecystokinin (CCK)a metabolic hormone best known for its roles in satiety regulation and fat metabolism-is increased in the lungs of obese mice and that pharmacological blockade of CCK and its receptor (CCKAR) signaling in the lung abolishes obesity-associated airway hyperresponsiveness (AHR)-a hallmark of asthma. By mining existing RNA-seq transcriptomic data, we first discovered CCKAR as a highly expressed G-protein-coupled receptor in primary human airway smooth muscle (ASM) cells. Interestingly, CCK is also expressed in ASM cells and is induced by free fatty acids. Activation of CCKAR by CCK induces ASM stiffening and contraction, which is abolished by either CRISPR-mediated CCKAR inactivation or CCKAR antagonists. In vivo, CCK levels are elevated in the lung of both genetically obese $(\mathrm{db} / \mathrm{db})$ and diet-induced obese mice. Importantly, intranasal administration of highly potent CCKAR antagonists (proglumide and devazepide) abolishes AHR in both genetically obese and diet-induced obese mice. Together, our results reveal an unexpected role for the metabolic hormone CCK and its receptor CCKAR in airway smooth muscle cells and in obesity-associated asthma. Our study provides critical pre-clinical data that support the repurposing of CCKAR antagonists as a novel therapy for obese asthmatics.

\section{Introduction}

Asthma is a major chronic lung disease that affects over 300 million people worldwide ${ }^{1}$. A key feature of asthma is airflow limitation, often caused by hyperactive contraction of airway smooth muscle (ASM) cells $^{2}$. Consequently, a major therapeutic goal for asthma is to reduce ASM constriction ${ }^{2}$. $\beta$-agonists-a mainstay asthma therapy-work by relaxing ASM to relieve bronchospasm ${ }^{3,4}$. However, long-term use of $\beta$-agonists, especially long-acting $\beta$-agonists (LABAs), can lead to paradoxically adverse effects, including loss of asthma control and even death ${ }^{5-7}$. Furthermore, standard controller therapy that uses inhaled corticosteroid in combination with a $\beta$-agonist does not work well for certain patient populations, notably obese asthmatics.

Obesity is a well-known risk factor for asthma: obesity increases both the prevalence and incidence of asthma and worsens asthma control ${ }^{8-12}$. It is estimated that in the US alone, obesity leads to approximately 250,000 new cases of asthma each year ${ }^{11}$. In general, obese asthmatics have more symptoms, use more healthcare, and have worse quality of life ${ }^{12,13}$. Consistent with these human studies, obese mice exhibit innate airway hyperresponsiveness $(A H R)^{14-17}$, a cardinal feature of asthma. Obesity also affects response to asthma therapy ${ }^{10,18,19}$. For example, corticosteroids are less effective in obese patients ${ }^{18-21}$, in whom asthma control is more difficult to achieve ${ }^{13,22-24}$. There is also some evidence that bronchodilators are less effective in obese asthmatics ${ }^{25,26}$. Despite the overwhelming evidence linking obesity to worsened asthma and to reduced response to therapy, the underlying mechanisms are not well understood. Consequently, for the obese asthmatic there are few therapeutic options. 
Cholecystokinin (CCK) is a peptide hormone found predominantly in the gastrointestinal ( $\mathrm{Gl}$ ) tract and throughout the central nervous system (CNS) ${ }^{27}$. In the GI tract, CCK is released in response to food intake to regulate the motility and contraction of gall bladder and stomach ${ }^{28,29}$. In the CNS, CCK acts as a neurotransmitter to regulate satiety and nociception ${ }^{30}$. CCK has two specific receptors: CCKAR and $\mathrm{CCKBR}^{31,32}$. CCKAR mediates most of the CCK's action in the GI tract and in the inhibition of food intake, whereas CCKBR plays a more prominent role in the CNS by regulating anxiety and neuroleptic activity ${ }^{33}$. In the GI tract, CCKAR induces the contraction of smooth muscle of gall bladder and stomach ${ }^{34-36}$. Intriguingly, Stretton and Barnes showed in a 1989 study that a CCK octapeptide constricts both guinea pig and human airways ${ }^{37}$. However, the potential in vivo function of CCK in the lung was not further explored, and it was not known which CCK receptor mediates the CCK action in the airway.

Here, we present evidence that CCK and its receptor CCKAR are functionally expressed in and induce contraction of airway smooth muscle cells. In vivo, CCK is upregulated in the respiratory system of dietinduced obese and genetically obese mice which suggests that it contributes to the heightened AHR associated with obesity. Our data using obese mouse models further demonstrate that antagonizing CCK/CCKAR in the lung abolishes obesity-induced AHR and suggest the possibility of developing CCKAR antagonists as novel therapeutic strategies for asthma in the obese.

\section{Results}

CCKAR expression in ASM. A large family of proteins known as G-protein-coupled receptors (GPCR) are direct targets of roughly $35 \%$ of all modern pharmaceuticals ${ }^{38,39}$. Many existing asthma drugs such as $\beta$ agonists, anti-cholinergics, and leukotrienes work by targeting their cognate GPCRs in the airways ${ }^{40}$. In order to identify potential new therapeutic targets for asthma, we evaluated the expression of nonolfactory GPCRs (361 in total) in primary human ASM cells using our previously published RNA-seqbased transcriptomic dataset ${ }^{41}$. We assessed the expression of each non-olfactory GPCR based on the values of Fragments Per Kilobase of transcript per Million mapped reads (FPKM). From this analysis, we identified $115(\sim 32 \%)$ non-olfactory GPCRs that are moderately-to-highly expressed (FPKM > 0.1) in ASM cells (Supplementary Table).

Among the top 5 expressed GPCRs, CCKAR has not been previously implicated in any aspect of airway smooth muscle function ${ }^{42-47}$. CCKAR expression in ASM cells has a high FPKM value of 26.52. In comparison, the $\beta$-adrenergic receptor 2 (gene name ADRB2), which is the target of bronchodilator $\beta$ agonists, has an FPKM value of 0.27 (Supplementary Table). Interestingly, the other CCK receptor CCKBR is not expressed at all in the ASM cells (no reads in the RNAseq data). The expression and function of CCKAR have been mostly investigated in extrapulmonary tissues such as the gallbladder and the

sphincter of Oddi where it mediates CCK-induced contraction and relaxation, respectively ${ }^{48,49}$. However, CCKAR's surprising high expression in ASM cells suggests a potential biological role for the receptor in the ASM and airways. 
We next validated the expression of CCKAR in ASM cells using qRT-PCR analysis. We found that CCKAR is highly expressed in ASM cells but is very lowly expressed in other cell types of airways such as the lung fibroblasts and is absent in both basal and differentiated bronchial epithelial cells (Fig. 1a). Consistent with the RNAseq data, we did not detect CCKBR expression in ASM cells using qRT-PCR (data not shown). Immunostaining of human airway tissue sections revealed co-localization of CCKAR with a-SMA, a widely known marker of airway smooth muscle (Fig. 1b). Together these results demonstrate the expression of CCKAR in ASM cells.

Activation of CCKAR evokes ASM contraction. CCKAR is known to mediate smooth muscle contraction in gallbladder and pyloric sphincter; however, in the sphincter of Oddi, CCKAR mediates relaxation of the muscle tissue. To test whether CCKAR evokes contraction or relaxation in ASM cells, we measured changes of cell stiffness using a biomechanical assay known as optical magnetic twisting cytometry $(\mathrm{OMTC})^{50}$. Changes in cell stiffness measured in this way are tightly related to changes in contractile force..$^{51}$ Upon treatment with the highly specific CCKAR agonist A71623 (10 nM) the stiffness of ASM cells increased (Fig. 1C). Such increases in cell stiffness are comparable in magnitude and timing ( 1.4 fold, $\sim 50$ seconds) to those induced by acetylcholine, a known ASM constrictor, and opposite to those induced by of albuterol, a $\beta$-agonist that relaxes ASM cells. To assess more directly the effect of A71623 on the contractility of ASM cells, we used traction force microscopy (TFM); TFM measures the contractile forces exerted by cells on their surroundings (e.g. extracellular matrix) ${ }^{52}$, to directly assess the effect of A71623 on the contractility of ASM cells. Using TFM, we measured contractile force in ASM cells pre- and post-addition of A71623 and found that A71623 increased ASM cell contraction as shown by the 2-fold increase in the force response ratio compared to control (Fig. 1d). Together these biophysical measurements demonstrated that the CCKAR agonist A71623 induces contraction of ASM cells.

We next investigated whether ASM contraction by A71623 is specifically mediated by CCKAR using either genetic or pharmacologic interventions. Using the CRISPR-Cas9 technology, we first generated independent CCKAR-knockout lines (CCKAR-KO1 and CCKAR-KO2) using two different guide RNAs targeting distinct regions of the CCKAR gene (Fig. 2a). CRISPR-mediated knockout of CCKAR prevented A71623-induced contraction of ASM cells as measured by TFM (Fig. 2b). Similarly, there was no increase in cell stiffness upon A71623 treatment in the two CCKAR-KO ASM cell lines (Fig. 2c). Consistent with the results in CCKAR-KO cells, pretreatment with a pan-CCK antagonist lorglumide (Fig. 2d) or a highly specific CCKAR antagonist devazepide (Fig. 2e) completely abolished A71623-induced stiffening of ASM cells. Together, these data demonstrate that CCKAR is required for CCK agonist-induced contraction in ASM cells.

CCK expression and induction by free fatty acids in ASM cells. Our in vitro biophysical experiments so far have used a synthetic tetrapeptide analogue of CCK hormone (A71623) that was given exogenously to ASM cell cultures. Studies have shown that cells that express CCK receptors often also express endogenous CCK which provides evidence for autocrine activation and signaling of CCKAR ${ }^{53-55}$. We thus examined whether ASM cells also express CCK. Our qRT-PCR measurements (Fg.3a) showed that CCK is expressed in ASM cells at a level much higher than in other airway cells such as lung fibroblasts, basal 
epithelial cells, or differentiated epithelial cells. Immunostaining experiment also showed that CCK is expressed in the ASM cells (marked by a-SMA staining) in the mouse airways (Fig. 3b).

As the ligand binding site of CCKAR is located extracellularly ${ }^{56}$, we asked whether endogenous CCK is secreted by ASM cells. In vivo, the CCK hormone is induced and secreted by cells lining the small intestine in response to ingestion of food, especially those with high fat content ${ }^{57}$. Because free fatty acids have been shown to induce CCK secretion in culture ${ }^{57}$ and in vivo ${ }^{58}$, we next sought to determine if CCK may likewise be induced by free fatty acids in ASM cells. We treated ASM cells with various types of free fatty acids and assessed their effects on CCK expression and secretion. As shown in Fig. 3c, treatment with long-chain free fatty acids (palmitoleic and myristoleic acids) increased CCK mRNA by 3.5 fold in ASM cells, whereas treatment with free fatty acids with shorter carbon chains (hexanoic acid, decenoic acid, dodecanoic acid) did not significantly increase CCK mRNA expression. Consistent with the mRNA expression data, the CCK hormone level as detected by ELISA increased by $\sim 30$ and $\sim 15$ fold in ASM cells treated with palmitoleic and myristoleic acids (Fig. 3d). Together these data show that expression of CCK hormone is induced by free fatty acids in ASM cells.

Elevated CCK levels in the lungs of obese mice. Because high levels of circulating free fatty acids are associated with obesity and obesity-associated $A \mathrm{HR}^{59-61}$, and because our data revealed that fatty acids induce CCK expression in ASM cells, we asked whether CCK expression is elevated concomitantly with an increased level of fatty acids in vivo. We examined the lungs of both high-fat-diet (HFD)-induced obese and genetically obese $(d b / d b)$ mice. We found that the level of free fatty acids (FFA) in the bronchioalveolar lavage (BAL) fluid of HFD-fed obese mice was elevated by at least 1.9-fold compared to regular chow-fed lean mice (Fig. 3e). Importantly, there was a concomitant increase in CCK mRNA in the lungs of HFD-fed mice compared to their controls (Fig. 3f), whereas the expression of CCKAR mRNA in HFD-fed obese mice was not significantly changed (Supplementary Fig. S2a). Similarly, we also found that the level of FFA was higher ( 1.5 fold) in the BAL of $d b / d b$ mice than in wild-type controls (Fig. 3g). Moreover, the expression of CCK mRNA was increased by $~ 1.4$ fold in $d b / d b$ mice compared to wild type controls (Fig. 3h). Together these data reveal an elevated CCK level in the lungs of obese mice.

Antagonizing CCK/CCKAR abolishes the innate AHR in obese mice. Obese mice exhibit innate AHR, a clinical feature of asthma ${ }^{62}$. Our data showing elevated CCK in obese lungs suggest that an increased CCK/CCKAR signaling and subsequent ASM contraction may contribute to the development of obesityassociated AHR. We therefore directly tested whether antagonizing CCKAR counteracts AHR in obese mouse models. Mice fed with high-fat diet $(60 \% \mathrm{kcal} / \mathrm{fat})$ gain weight very rapidly and develop AHR, which becomes pronounced at 21-24th weeks of age compared to the regular chow-fed, lean controls ${ }^{15}$. We administered CCKAR antagonists into HFD-obese and control mice and then assessed AHR in response to increasing doses of methacholine (Fig. 4a). Intranasal administration of proglumide, a potent CCKAR antagonist, reduced AHR in HFD-fed obese mice as shown by the decrease in pulmonary resistance $\left(R_{L}\right)$ to a level similar to that of lean controls (Fig. $4 b$ ). Similarly, intranasal treatment with devazepide, another potent and highly selective CCKAR antagonist, significantly reduced the innate AHR 
of HFD-fed obese mice as compared to vehicle-treated obese mice (Fig. 4c). Because previous reports have suggested that the AHR in obese mice is facilitated by the pro-inflammatory cytokine IL-17 $A^{63}$ and that obese asthmatics have increased levels of IL-17A ${ }^{64}$, we measured the expression of IL-17A in the lungs of proglumide and vehicle-treated mice. We found that while IL-17A is increased in HFD-fed obese mice compared to lean mice, CCKAR antagonist treatment did not alter the expression of IL-17A (Supplementary Fig. S4), suggesting that effect of CCK/CCKAR on AHR is independent of IL-17A production and likely of inflammation.

We likewise tested the effect of antagonizing CCKAR on the AHR of genetically induced obese mice. $D b / d b$ mice, which lack the receptor for leptin, rapidly develop obesity on the standard chow diet and exhibit AHR starting at 8 weeks, even in the absence of any inciting exposure ${ }^{15,64}$. Similar to the effect of antagonizing CCKAR in HFD-fed mice, administration of proglumide or devazepide significantly reduced the pulmonary resistance in response to methacholine in $d b / d b$ mice (Fig. $4 \mathrm{~d}-\mathrm{f}$ ).

\section{Discussion}

Obesity is a significant risk factor for the development of symptoms, worsening of those symptoms, and poor management of asthma ${ }^{8,21,65}$. The underlying mechanisms for these effects remain incompletely understood. The lack of concrete knowledge of the pathophysiology of obesity-associated asthma precludes the development of new therapies that could effectively treat asthma in obese patients. Our study uncovers a novel mechanism for obesity-associated asthma based on the largely unexplored ability of CCK hormone and its receptor CCKAR in promoting ASM contraction. Furthermore, our study provides direct pre-clinical evidence for the repurposing of potent CCKAR antagonists as novel, effective therapies for obese asthmatics.

Although CCK increases airway constriction ex vivo ${ }^{37}$, neither the identity of the cognate receptor nor the in vivo implication of such airway constriction was known. Our study first identified CCKAR as a highly expressed GPCR in ASM cells from an unbiased RNA sequencing dataset that led us to investigate its role in CCK-induced airway constriction. Using genetic ablation and pharmacologic intervention, we firmly established the role of CCK and CCKAR in contracting ASM cells. We also found that CCK is elevated in the lungs of mouse models of obesity-associated asthma which implied that CCK/CCKAR mediates AHR in the obese. Indeed, blocking CCKAR using potent CCKAR antagonists abolishes obesity associated with AHR in two different obese mouse models. Our results support a hypothesis in which elevated CCK level associated with obesity increases CCKAR signaling in the lung to constrict ASM cells to drive bronchoconstriction and AHR. Antagonizing such elevated CCK/CCKAR signaling in the ASM blocks obesity-induced AHR and thus presents a completely novel way to treat obese asthmatics (Fig. $4 \mathrm{~g}$ ).

Our studies clearly established the in vivo role of CCK-induced airway constriction as blocking the heightened CCK/CCKAR signaling using potent antagonists decreased the pulmonary resistance to methacholine challenge in two distinct mouse models of obesity-associated asthma - a genetically obese mouse model and a diet-induced mouse model. CCKAR antagonists are intended for gastric ulcer 
to inhibit gastrointestinal motility and gastric secretions, and are known to possess a favorable safety profile ${ }^{66-69}$. Thus, our data showing that CCKAR antagonists attenuated AHR support the idea that these drugs could potentially be repurposed for the treatment of asthma in the obese. We used two antagonists (proglumide and devazepide) possessing different selectivity profiles with respect to CCK receptors. Devazepide, in addition to being more potent in reducing AHR, is also the more highly selective CCKAR antagonist than the pan-CCK receptor-targeting proglumide. Thus, the use of devazepide or other highly selective CCKAR antagonists would be the priority for asthma drug repurposing in order to limit the undesirable effects brought by the possibility of activating the other CCK receptor (CCKBR). CCKAR antagonists are normally administered via oral or intraperitoneal delivery that increases drug bioavailability in the systemic circulation. We showed that intranasal administration of CCKAR antagonists is sufficient to reduce AHR in obese mice. By this method, the potential unwanted effects of CCKAR inhibition in extra-pulmonary tissues is likely lower, although we did not examine the toxicity profile of intranasal administration compared to other modes of delivery. Potent bronchodilators are commonly delivered via nebulization suggesting that CCKAR antagonists may be developed in similar formulation to effectively abrogate asthma symptoms while avoiding undesirable systemic effects.

Our experimental results suggest an autocrine stimulatory loop of CCK-activated, CCKAR-mediated ASM contraction where free fatty acids induce the expression and secretion of CCK in ASM cells. However, such activation of CCKAR signaling in ASM may also come from circulating CCK that reach the lung given that plasma levels of CCK are elevated in obese rodents or after high-fat diet intake ${ }^{70-73}$. Individuals with high circulating CCK levels may thus be prone to altered lung function and heightened AHR. Similarly, conditions that lead to elevation of plasma CCK may trigger asthma symptoms. It was reported that exercise elevates plasma CCK levels in individuals with exercise-induced asthma ${ }^{74}$. Similarly, plasma CCK was observed to be increased by high altitude ${ }^{75}$. It is possible that the worsening of asthma symptoms associated with these clinical scenarios is related to elevated plasma CCK levels and may be alleviated by blocking CCK/CCKAR signaling with the antagonists.

Our data suggest that, at least in obesity-associated asthma, the heightened CCK/CCKAR signaling in the lung appears to lead to increased ASM contractility that is independent of airway inflammation as CCKAR antagonists did not alter IL-17A, a pro-inflammatory cytokine that has been shown to mediate AHR in obesity-associated mouse models. This is supported by our in vitro biophysical studies which demonstrated that CCK causes direct ASM contraction. While our studies focused largely on inhibiting CCKAR-mediated contraction and did not extensively study the effect of CCKAR antagonists on airway inflammation, which is thought to be an important precipitating factor in asthma, our data showing the absence of effect of CCKAR antagonists on IL-17A nevertheless suggest that blocking CCKAR-mediated ASM contraction is sufficient to abolish obesity-associated AHR.

In summary, there is a pressing need to develop new therapeutic strategies for asthma especially for the obese asthmatic subpopulation. Our present study reveals that CCKAR mediates CCK-induced contraction of ASM cells and that CCK/CCKAR signaling is a novel mechanism underlying obesity-induced AHR. Such findings imply that blocking CCKAR may be developed into a new asthma therapy for obese asthmatic 
patients who still rely on ineffective symptomatic treatments mainly prescribed for lean asthmatics. Our study provides critical pre-clinical evidence for repurposing CCK/CCKAR antagonists, many of them already developed for gastrointestinal diseases ${ }^{62}$, to treat obesity-associated asthma. Future clinical studies built upon our findings here may lead to the development of much-needed effective therapies for obese asthmatics.

\section{Methods}

\section{Cell Culture}

Primary cultures of human ASM cells were provided by Dr. Reynold Panettieri (Rutgers University) and cultured as described ${ }^{76}$. ASM cultures were maintained in F12 (HAM) nutrient medium (Gibco) supplemented with $10 \%$ FBS, 100 units $/ \mathrm{ml}$ penicillin, $100 \mu \mathrm{g} / \mathrm{ml}$ streptomycin, $300 \mu \mathrm{g} / \mathrm{ml}$ L-glutamine, 4.8 mM HEPES, $0.34 \mathrm{mM} \mathrm{CaCl} 2$, and $2.4 \mathrm{mM} \mathrm{NaOH}$. Passages 3-8 were used in the experiments.

Normal human bronchial epithelial (HBE) cells were provided by Dr. Jin-ah Park (HSPH). They were obtained from the Marsico Lung Institute/Cystic Fibrosis Center at the University of North Carolina, Chapel Hill and cultured in bronchial epithelial basal media (Lonza) supplemented with bovine pituitary extract $(52 \mathrm{mg} / \mathrm{mL})$, hydrocortisone $(0.5 \mathrm{mg} / \mathrm{mL})$, human epidermal growth factor $(25 \mathrm{ng} / \mathrm{mL})$, epinephrine $(0.5 \mathrm{mg} / \mathrm{mL})$, insulin $(5 \mathrm{mg} / \mathrm{mL})$, triiodothyronine $(6.5 \mathrm{ng} / \mathrm{mL})$, transferrin $(10 \mathrm{mg} / \mathrm{mL})$, gentamicin $(50 \mathrm{mg} / \mathrm{mL})$, amphotericin-B $(50 \mathrm{ng} / \mathrm{mL}), \mathrm{BSA}(1.5 \mathrm{mg} / \mathrm{mL})$, andretinoic acid $(50 \mathrm{nM})$. To establish ALI culture, HBE cells were fed with a 1:1 mixture of bronchial epithelial basal media and DMEM (Mediatech, Tewksbury, MA) supplemented with the same components above except that the concentration of human epidermal growth factor was reduced $(0.5 \mathrm{ng} / \mathrm{mL})$. Cells were cultured on collagen-coated 12-Transwell plate (Corning, Tewksbury, MA) under submerged conditions. When the cells reached confluence, the medium was removed from the apical surface. ALI culture was maintained up to 14 days until needed ${ }^{77}$.

\section{Quantitative Real-Time (qRT)PCR}

Total RNA was extracted using the RNEasy kit according to the manufacturer's instructions (Qiagen, Cat. 74106). RNA was then reverse-transcribed using the Superscript III First-Strand Synthesis System (Life Technologies, Cat. 18080-051). Quantitative PCR was performed using SYBR green master mix (Qiagen, Cat. 214145) with gene-specific primers

Human CCKAR forward: 5'- TGCTCAAGGATTTCATCTTCG

Human CCKAR reverse: 5'- TGGTCACCAGATTAAAGGTAGATACA

Human CCK forward: 5'- CTGGCAAGATACATCCAGCA

Human CCK reverse: 5'- CCATGTAGTCCCGGTCACTT

Human ACTB ( $\beta$-actin) forward: 5'- CCAACCGCGAGAAGATGA 
Human ACTB ( $\beta$-actin) reverse: 5'- CCAGAGGCGTACAGGGATAG

Human 18s rRNA forward: 5'- CCGATTGGATGGTTTAGTGAG

Human 18s rRNA reverse: 5' - AGTTCGACCGTCTTCTCAGC

Mouse CCK forward: 5'- GCTGATTTCCCCATCCAAA

Mouse CCK reverse: 5'- GCTTCTGCAGGGACTACCG

Mouse CCKAR forward: 5'- GATGCCAGCCAGAAGAAATC

Mouse CCKAR reverse: 5'- ACAGCCATCGCTATCCTCAT

Mouse IL-17A forward: 5'- CAGGGAGAGCTTCATCTGTGT

Mouse IL-17A reverse: 5'- GCTGAGCTTTGAGGGATGAT

Mouse ACTB ( $\beta$-actin) forward: 5'- CTAAGGCCAACCGTGAAAAG

Mouse ACTB ( $\beta$-actin) reverse: 5'- ACCAGAGGCATACAGGGACA

Immunofluorescence Staining: Immunofluorescence staining of lung tissue sections were performed as previously described ${ }^{54}$. Antigen retrieval was performed using $10 \mathrm{mM}$ citrate buffer, $\mathrm{pH}$ 6.0. Lung tissue sections were blocked with PBS supplemented with $5 \%$ normal donkey serum and $0.2 \%$ Triton X-100 for 1 hour at room temperature. Primary antibodies (1:1000) used were anti-CCKAR (Pierce, Cat. PA3-116), antiCCK (Santa Cruz Biotechnology, Inc., Cat. Sc-21617), and anti-a-SMA (Sigma, Cat. C6198). ProLong gold anti-fade mountant with DAPI (Life Technologies, Cat. P36941) was used for mounting samples.

Confocal images were taken using Leica SPE Confocal Microscope and the images were processed using ImageJ.

Generation of CCKAR-Knockout Cells: Two CCKAR-knockout cell lines (KO1 and KO2) were generated using CRISPR with the following guide RNAs (gRNAs): TAGAAAGCGGCACATATTCG for KO1 and CCGCTTGTTCCGAATCAGCA for KO2. Guides were cloned into lentiCRISPRv2 vector containing hSpCas9 cassette (Addgene) as previously described ${ }^{55}$. Briefly, oligonucleotides targeting the site sequence were synthesized with 3-bp NGG PAM sequence flanking the 3' end, annealed and cloned into the BsmBIdigested lentiCRISPRv2 vector. The resulting plasmids were transformed into Stbl3 bacteria (ThermoFisher Scientific, Cat. C737303) and purified using Mini-prep Kit (Qiagen, Cat. 27104).

Lentiviruses were produced by co-transfecting the lentiCRISPRv2 containing gRNAs with the packaging plasmids pVSVg and psPAX2 (AddGene) in HEK293T cells. Lentiviral transduction in HASM cells was performed in the presence of polybrene $(8 \mu \mathrm{g} / \mathrm{ul})$ for 24 hours. Stably transduced HASM cells were selected using puromycin (ThermoFisher Scientific, Cat. A1113803). T7E1 assay was performed to determine knockout efficiency. For the T7E1 assay, the genomic region harboring the target of gRNAs was first PCR amplified, subjected to denaturing and reannealing temperatures $\left(95^{\circ} \mathrm{C}\right.$ for $2 \mathrm{~min}$, ramp down at 
$-2^{\circ} \mathrm{C} / \mathrm{s}$ to $85^{\circ} \mathrm{C}$, ramp down at $-0.1^{\circ} \mathrm{C} / \mathrm{s}$ to $25^{\circ} \mathrm{C}$, and stopped at $16^{\circ} \mathrm{C}$. T7E1 (New England Biolabs, Cat. M0302S) cleavage reaction was then performed at $37^{\circ} \mathrm{C}$ for $20 \mathrm{~min}$. The PCR products were visualized using $1.5 \%$ agarose gel.

\section{Optical Magnetic Twisting Cytometry}

Cell stiffness was measured using optical magnetic twisting cytometry as described ${ }^{50}$. Briefly, ASM cells were cultured to confluence in collagen-coated 96-well plate (Stripwell Microplates, Corning, NY). After overnight serum starvation, cells were incubated with ferrimagnetic beads $(4.5 \mu \mathrm{m}$ in diameter, produced in house, and coated with poly-L-lysine) for $20 \mathrm{~min}$ to allow beads to attach to cell surfaces ${ }^{78}$. The beads were then magnetized with a strong magnetic pulse in the horizontal direction and twisted with a much weaker oscillatory magnetic field $(0.77 \mathrm{~Hz})$ in the vertical direction. The ratio of magnetic torque to bead motion ${ }^{50,78,79}$ was used to determine cell stiffness and average of stiffness normalized to baseline to determine relative changes in cell stiffness after drug treatment.

\section{Traction Force Microscopy}

Cell contraction was measured using traction force microscopy as described ${ }^{52}$. Briefly, ASM cells were cultured to confluence in 96-well plates containing custom elastic polyacrylamide substrate. The average contractile stress was measured before plating the cells (reference), prior to treatment (baseline) and 1 hour after addition of drugs (treatment). Data are reported as the force-response ratio of treatment vs. baseline contraction.

\section{CCK Enzyme Immunoassay}

We used a CCK Enzyme Immunoassay Kit (Sigma-Aldrich, Cat RAB0039) that is based on the principle of competitive enzyme immunoassay. Samples and standards were mixed with biotinylated CCK (final concentration was $20 \mathrm{pg} / \mathrm{mL}$ in every sample). A 96-well plate was coated with anti-CCK antibody and incubated overnight at $4^{\circ} \mathrm{C}$ with gentle shaking (1-2 cycles/sec). Following incubation, the wells were washed 5 times with wash buffer. Samples and standards were added into appropriate wells. A blank well containing assay diluent was also included. The plate was incubated overnight at $4^{\circ} \mathrm{C}$ with gentle shaking (1-2 cycles/sec). Following incubation, the wells were washed 5 times with wash buffer. A solution of HRP-streptavidin was added to each well and the plate was incubated for 45 minutes with gentle shaking at room temperature. The solution was discarded and the plate washed 5 times with wash buffer. TMB One-Step Substrate Reagent was then added to each well and the plate was incubated for 30 minutes at room temperature in the dark with gentle shaking. Following incubation, a stop solution was added to each well and absorbance was read at $450 \mathrm{~nm}$ (SpectraMax 190 Microplate Reader, Molecular Devices). A four-parameter logistic regression model was then used to plot the standard curve and to calculate the concentration of CCK in the sample.

\section{Free Fatty Acid Quantification Assay}


For the detection of FFA in samples, the Free Fatty Acid Quantification Kit (Biovision, Cat. K612-100) was used. This assay converts fatty acids into their CoA-derivatives which are then oxidized with the concomitant generation of color or fluoresence. Fifty (50) $\mu$ l of samples (dissolved in supplied assay buffer if needed) were added to each well in a 96-well plate. The acyl-CoA synthesis was then performed by adding $2 \mu \mathrm{l}$ of ACS reagent into the wells, mixed, and incubated at $37^{\circ} \mathrm{C}$ for 30 minutes. A reaction mix (44 $\mu$ l assay buffer, $2 \mu \mathrm{l}$ fatty acid probe, $2 \mu$ l enzyme mix, and $2 \mu \mathrm{l}$ enhancer was added to each well and incubated at $37^{\circ} \mathrm{C}$ for 30 minutes in the dark. After incubation the absorbance was measured at $570 \mathrm{~nm}$ (SpectraMax 190 Microplate Reader, Molecular Devices). A standard curve was generated using palmitic acid and the concentration of fatty acid was determined using formula, [FFA] $=\mathrm{Fa} / \mathrm{Sv}(\mathrm{nmol} / \mu \mathrm{l}$ or $\mathrm{mM})$ where $\mathrm{Fa}$ is the fatty acid amount in the well obtained from the standard curve and Sv is the sample volume $(\mu \mathrm{l})$ added to the sample well.

\section{Mouse models of obesity-associated AHR}

This study was approved by the Harvard Medical Area Standing Committee on Animals. Obese mice and their corresponding lean controls were acquired from the Jackson Laboratory. For the development of diet-induced obesity, male C57BL/6J mice were placed on a high fat diet where $60 \%$ of calories were derived from fat in the form of lard (D12492, Research Diets) starting at 8 weeks of age for 13-14 weeks until the time of the experiment. Mice places on a regular chow diet (PicoLab 5053, Lab Diets) served as corresponding lean controls. Mice fed with HFD gain weight very rapidly and develop AHR compared to their corresponding lean controls, which are fed with regular chow diet and have normal airway reactivity ${ }^{15,64}$. $D b / d b$ mice, which lack the long form of the leptin receptor, were used as the mouse model of genetic obesity. $D b / d b$ mice are substantially obese compared to the age- and sex-matched wild type controls and exhibit increased airway responsiveness to methacholine even in the absence of any inciting stimuli (e.g. ovalbumin challenge) ${ }^{80}$.

\section{Measurement of Airway Hyperresponsiveness (AHR)}

This study was approved by the Harvard Medical Area Standing Committee on Animals. The forcedoscillation technique was used to assess AHR using the Flexivent machine (Scireq, Montreal, QC, Canada). Mice were first anesthetized with xylazine $(7 \mathrm{mg} / \mathrm{kg})$ and sodium pentobarbital $(50 \mathrm{mg} / \mathrm{kg})$ and an incision along the tracheal wall was made to expose the trachea. The trachea was then cannulated with a tubing adaptor and then connected to the machine. The mice were ventilated and instrumented for the measurement of pulmonary mechanics and airway responsiveness in response to increasing doses of methacholine ${ }^{81}$. The chest wall was opened to expose the lungs to atmosphere pressure and a positive end expiratory pressure of $3 \mathrm{~cm} \mathrm{H}_{2} \mathrm{O}$ was applied. Obese mice were ventilated at a respiratory rate of 180 breaths/minute while lean mice were ventilated at 150 breaths/min. Changes in pulmonary resistance $\left(R_{L}\right)$ were assessed at the baseline and after delivery of aerosolized PBS and aerosolized methacholine in increasing doses.

\section{Statistical Analysis}


Data are given as mean \pm SEM. Differences between groups were analyzed by t-test or two-way ANOVA with Bonferroni's multiple comparisons (Prism v8; GraphPad Software Inc). A $p<0.05$ (two-tailed) was considered to be statistically significant.

Data Availability: GPCR expression data are from published datasets (ref.41) that are already deposited in public databases available at the Gene Expression Omnibus Web site (http://www.ncbi.nlm.nih.gov/geo/) under accession GSE52778.

\section{Declarations}

\section{Acknowledgements}

This work was supported mainly by a scholar award from the American Asthma Foundation to Q.L.

Author Contributions: R.A.P. and Q.L. conceived the project and designed the experiments. R.A.P. performed most of the experiments. Q.L. supervised the project. C.Y.P and J.F. contributed to the biomechanical assays and analyses. M.S., D.K., and S.A.S. contributed to the AHR and analyses. E.I. and M.B.H contributed to the immunostaining. B.H., A.K., S.T.W., and K.L. contributed to the RNA-Seq analyses. R.A.P. and Q.L. wrote the paper with input from other co-authors.

Competing Interests: Q.L. is co-founder, scientific advisor and shareholder of Vesigen Therapeutics. The other authors declare no competing interests.

\section{References}

1. 2020 GINA Main Report. Global Initiative for Asthma - GINA https://ginasthma.org/gina-reports/.

2. Doeing, D. C. \& Solway, J. Airway smooth muscle in the pathophysiology and treatment of asthma. $J$ Appl Physiol (1985) 114, 834-843 (2013).

3. Johnson, M. The $\beta$-Adrenoceptor. Am J Respir Crit Care Med 158, S146-S153 (1998).

4. McGraw, D. W. \& Liggett, S. B. Molecular mechanisms of beta2-adrenergic receptor function and regulation. Proc Am Thorac Soc 2, 292-296; discussion 311-312 (2005).

5. Cockcroft, D. W. \& Sears, M. R. Are inhaled longacting $\beta 2$ agonists detrimental to asthma? Lancet Respir Med 1, 339-346 (2013).

6. Lipworth, B. J. Airway Subsensitivity with Long-Acting ß2-Agonists. Drug-Safety 16, 295-308 (1997).

7. Mansfield, L. E. The future of the long-acting beta-adrenergic bronchodilators in the treatment of asthma. Allergy Asthma Proc 29, 103-108 (2008).

8. Ford, E. S. The epidemiology of obesity and asthma. J Allergy Clin Immunol 115, 897-909; quiz 910 (2005).

9. Shore, S. A. Obesity and asthma: implications for treatment. Curr Opin Pulm Med 13, 56-62 (2007). 
10. Mosen, D. M., Schatz, M., Magid, D. J. \& Camargo, C. A. The relationship between obesity and asthma severity and control in adults. J Allergy Clin Immunol 122, 507-511.e6 (2008).

11. Beuther, D. A. \& Sutherland, E. R. Overweight, obesity, and incident asthma: a meta-analysis of prospective epidemiologic studies. Am J Respir Crit Care Med 175, 661-666 (2007).

12. Sutherland, E. R. Linking obesity and asthma. Ann N Y Acad Sci 1311, 31-41 (2014).

13. Lavoie, K. L., Bacon, S. L., Labrecque, M., Cartier, A. \& Ditto, B. Higher BMI is associated with worse asthma control and quality of life but not asthma severity. Respir Med 100, 648-657 (2006).

14. Johnston, R. A., Theman, T. A. \& Shore, S. A. Augmented responses to ozone in obese carboxypeptidase E-deficient mice. Am J Physiol Regul Integr Comp Physiol 290, R126-133 (2006).

15. Johnston, R. A. et al. Diet-induced obesity causes innate airway hyperresponsiveness to methacholine and enhances ozone-induced pulmonary inflammation. J Appl Physiol (1985) 104, 1727-1735 (2008).

16. Lu, F. L. et al. Increased pulmonary responses to acute ozone exposure in obese $\mathrm{db} / \mathrm{db} \mathrm{mice}$. Am J Physiol Lung Cell Mol Physiol 290, L856-865 (2006).

17. Shore, S. A., Rivera-Sanchez, Y. M., Schwartzman, I. N. \& Johnston, R. A. Responses to ozone are increased in obese mice. J Appl Physiol (1985) 95, 938-945 (2003).

18. Peters-Golden, M. et al. Influence of body mass index on the response to asthma controller agents. Eur Respir J 27, 495-503 (2006).

19. Boulet, L.-P. \& Franssen, E. Influence of obesity on response to fluticasone with or without salmeterol in moderate asthma. Respir Med 101, 2240-2247 (2007).

20. Camargo, C. A. et al. Body mass index and response to asthma therapy: fluticasone propionate/salmeterol versus montelukast. J Asthma 47, 76-82 (2010).

21. Sutherland, E. R., Goleva, E., Strand, M., Beuther, D. A. \& Leung, D. Y. M. Body mass and glucocorticoid response in asthma. Am J Respir Crit Care Med 178, 682-687 (2008).

22. Dixon, A. E. et al. Effect of obesity on clinical presentation and response to treatment in asthma. $J$ Asthma 43, 553-558 (2006).

23. Dias-Júnior, S. A. et al. Effects of weight loss on asthma control in obese patients with severe asthma. Eur Respir J 43, 1368-1377 (2014).

24. van Leeuwen, J. C., Hoogstrate, M., Duiverman, E. J. \& Thio, B. J. Effects of dietary induced weight loss on exercise-induced bronchoconstriction in overweight and obese children. Pediatr Pulmonol 49, 1155-1161 (2014).

25. Wong, H. S., Ng, H. L., Kang, C., Mok, Y. J. \& Choo, X. N. Do obese asthmatics have a worse bronchodilator response? European Respiratory Journal 46, (2015).

26. McGarry, M. E. et al. Obesity and bronchodilator response in black and Hispanic children and adolescents with asthma. Chest 147, 1591-1598 (2015).

27. Chandra, R. \& Liddle, R. A. Cholecystokinin. Curr Opin Endocrinol Diabetes Obes 14, 63-67 (2007). 
28. Chaudhri, O., Small, C. \& Bloom, S. Gastrointestinal hormones regulating appetite. Philos Trans R Soc Lond B Biol Sci 361, 1187-1209 (2006).

29. Liddle, R. A. Cholecystokinin cells. Annu Rev Physiol 59, 221-242 (1997).

30. Beinfeld, M. C., Meyer, D. K. \& Brownstein, M. J. Cholecystokinin in the central nervous system. Peptides 2, 77-79 (1981).

31. Noble, F. et al. International Union of Pharmacology. XXI. Structure, distribution, and functions of cholecystokinin receptors. Pharmacol Rev 51, 745-781 (1999).

32. Wank, S. A. Cholecystokinin receptors. Am J Physiol 269, G628-646 (1995).

33. Kopin, A. S. et al. The cholecystokinin-A receptor mediates inhibition of food intake yet is not essential for the maintenance of body weight. J Clin Invest 103, 383-391 (1999).

34. de Weerth, A. et al. Characterization of CCK receptors in stomach smooth muscle: evidence for two subtypes. Biochimica et Biophysica Acta (BBA) - Biomembranes 1327, 213-221 (1997).

35. Si, X.-M., Huang, L., Paul, S. C., An, P. \& Luo, H.-S. Signal Transduction Pathways Mediating CCK-8SInduced Gastric Antral Smooth Muscle Contraction. DIG 73, 249-258 (2006).

36. Yu, P. et al. Signal transduction pathways mediating CCK-induced gallbladder muscle contraction. American Journal of Physiology-Gastrointestinal and Liver Physiology 275, G203-G211 (1998).

37. Stretton, C. D. \& Barnes, P. J. Cholecystokinin-octapeptide constricts guinea-pig and human airways. Br J Pharmacol 97, 675-682 (1989).

38. Sriram, K. \& Insel, P. A. G Protein-Coupled Receptors as Targets for Approved Drugs: How Many Targets and How Many Drugs? Mol Pharmaco/ 93, 251-258 (2018).

39. Hauser, A. S., Gloriam, D. E., Bräuner-Osborne, H. \& Foster, S. R. Novel approaches leading towards peptide GPCR de-orphanisation. Br J Pharmaco/ 177, 961-968 (2020).

40. Deshpande, D. A. \& Penn, R. B. Targeting G protein-coupled receptor signaling in asthma. Cell Signal $18,2105-2120$ (2006).

41. Himes, B. E. et al. RNA-Seq Transcriptome Profiling Identifies CRISPLD2 as a Glucocorticoid Responsive Gene that Modulates Cytokine Function in Airway Smooth Muscle Cells. PLOS ONE 9, e99625 (2014).

42. Kusser, B. et al. Polymorphisms in the bradykinin B2 receptor gene and childhood asthma. Biol Chem 382, 885-889 (2001).

43. Bertram, C. M. et al. Expression of kinin receptors on eosinophils: comparison of asthmatic patients and healthy subjects. J Leukoc Bio/ 85, 544-552 (2009).

44. Gong, D.-M. et al. Endothelial GPR124 Exaggerates the Pathogenesis of Atherosclerosis by Activating Inflammation. Cell Physiol Biochem 45, 547-557 (2018).

45. Chiu, L.-L., Perng, D.-W., Yu, C.-H., Su, S.-N. \& Chow, L.-P. Mold allergen, pen C 13, induces IL-8 expression in human airway epithelial cells by activating protease-activated receptor 1 and 2 . $J$ Immunol 178, 5237-5244 (2007). 
46. Reed, C. E. \& Kita, H. The role of protease activation of inflammation in allergic respiratory diseases. Journal of Allergy and Clinical Immunology 114, 997-1008 (2004).

47. Hall, R. J. et al. Functional genomics of GPR126 in airway smooth muscle and bronchial epithelial cells. The FASEB Journa/ 35, e21300 (2021).

48. Behar, J. \& Biancani, P. Effect of cholecystokinin and the octapeptide of cholecystokinin on the feline sphincter of Oddi and gallbladder. Mechanisms of action. J Clin Invest 66, 1231-1239 (1980).

49. Krishnamurthy, G. T. \& Krishnamurthy, S. Gallbladder, Sphincter of Oddi, Cholecystokinin, and Opioid Inter-Relationship. in Nuclear Hepatology: A Textbook of Hepatobiliary Diseases (eds. Krishnamurthy, G. T. \& Krishnamurthy, S.) 129-146 (Springer, 2000). doi:10.1007/978-3-662-22654-4_6.

50. Park, C. Y. et al. Mapping the cytoskeletal prestress. Am J Physiol Cell Physiol 298, C1245-1252 (2010).

51. Krishnan, R. et al. Reinforcement versus Fluidization in Cytoskeletal Mechanoresponsiveness. PLOS ONE 4, e5486 (2009).

52. Park, C. Y. et al. High-throughput screening for modulators of cellular contractile force. Integr Biol (Camb) 7, 1318-1324 (2015).

53. Heasley, L. E. Autocrine and paracrine signaling through neuropeptide receptors in human cancer. Oncogene 20, 1563-1569 (2001).

54. Herness, S. \& Zhao, F.-L. The neuropeptides CCK and NPY and the changing view of cell-to-cell communication in the taste bud. Physiol Behav 97, 581-591 (2009).

55. Oikonomou, E., Buchfelder, M. \& Adams, E. F. Cholecystokinin (CCK) and CCK receptor expression by human gliomas: Evidence for an autocrine/paracrine stimulatory loop. Neuropeptides 42, 255-265 (2008).

56. Silvente-Poirot, S., Escrieut, C. \& Wank, S. A. Role of the extracellular domains of the cholecystokinin receptor in agonist binding. Mol Pharmacol 54, 364-371 (1998).

57. McLaughlin, J. T. et al. Fatty acids stimulate cholecystokinin secretion via an acyl chain lengthspecific, Ca2+-dependent mechanism in the enteroendocrine cell line STC-1. J Physio/ 513 (Pt 1), 1118 (1998).

58. McLaughlin, J. et al. Fatty acid chain length determines cholecystokinin secretion and effect on human gastric motility. Gastroenterology 116, 46-53 (1999).

59. Reaven, G. M., Hollenbeck, C., Jeng, C. Y., Wu, M. S. \& Chen, Y. D. Measurement of plasma glucose, free fatty acid, lactate, and insulin for $24 \mathrm{~h}$ in patients with NIDDM. Diabetes 37, 1020-1024 (1988).

60. Boden, G. Obesity and free fatty acids. Endocrinol Metab Clin North Am 37, 635-646, viii-ix (2008).

61. Arner, P. \& Rydén, M. Fatty Acids, Obesity and Insulin Resistance. Obes Facts 8, 147-155 (2015).

62. Zhu, M. et al. Role of TNFR1 in the innate airway hyperresponsiveness of obese mice. J Appl Physiol (1985) 113, 1476-1485 (2012).

63. Marijsse, G. S. et al. Obese individuals with asthma preferentially have a high IL-5/IL-17A/IL-25 sputum inflammatory pattern. Am J Respir Crit Care Med 189, 1284-1285 (2014). 
64. Kim, H. Y. et al. Interleukin-17-producing innate lymphoid cells and the NLRP3 inflammasome facilitate obesity-associated airway hyperreactivity. Nat Med 20, 54-61 (2014).

65. Forno, E. \& Celedón, J. C. Predicting asthma exacerbations in children. Curr Opin Pulm Med 18, 6369 (2012).

66. Persiani, S. et al. Pharmacokinetics of dexloxiglumide after administration of single and repeat oral escalating doses in healthy young males. Int J Clin Pharmacol Ther 40, 198-206 (2002).

67. Herranz, R. Cholecystokinin antagonists: Pharmacological and therapeutic potential. Medicinal Research Reviews 23, 559-605 (2003).

68. Tucker, R. D. et al. A Cholecystokinin Receptor Antagonist Halts Nonalcoholic Steatohepatitis and Prevents Hepatocellular Carcinoma. Dig Dis Sci 65, 189-203 (2020).

69. Nadella, S. et al. Cholecystokinin Receptor Antagonist Therapy Decreases Inflammation and Fibrosis in Chronic Pancreatitis. Dig Dis Sci 65, 1376-1384 (2020).

70. Li, J., Ma, W. \& Wang, S. Slower gastric emptying in high-fat diet induced obese rats is associated with attenuated plasma ghrelin and elevated plasma leptin and cholecystokinin concentrations. Regul Pept 171, 53-57 (2011).

71. Matters, G. L. et al. Cholecystokinin Mediates Progression and Metastasis of Pancreatic Cancer Associated with Dietary Fat. Dig Dis Sci 59, 1180-1191 (2014).

72. Herrington, M. K. et al. Effects of high fat diet and cholecystokinin receptor blockade on pancreatic growth and tumor initiation in the hamster. Carcinogenesis 14, 1021-1026 (1993).

73. Degen, L., Matzinger, D., Drewe, J. \& Beglinger, C. The effect of cholecystokinin in controlling appetite and food intake in humans. Peptides 22, 1265-1269 (2001).

74. Hvidsten, D., Jenssen, T. G., Bolle, R. \& Burhol, P. G. Plasma gastrointestinal regulatory peptides in exercise-induced asthma. Eur J Respir Dis 68, 326-331 (1986).

75. Bailey, D. M. et al. Elevated plasma cholecystokinin at high altitude: metabolic implications for the anorexia of acute mountain sickness. High Alt Med Biol 1, 9-23 (2000).

76. Hu, R. et al. MicroRNA-10a controls airway smooth muscle cell proliferation via direct targeting of the PI3 kinase pathway. FASEB J 28, 2347-2357 (2014).

77. Panganiban, R. A. et al. A functional splice variant associated with decreased asthma risk abolishes the ability of gasdermin B to induce epithelial cell pyroptosis. J. Allergy Clin. Immunol. 142, 14691478.e2 (2018).

78. Zhou, E. H. et al. Universal behavior of the osmotically compressed cell and its analogy to the colloidal glass transition. Proc Natl Acad Sci U S A 106, 10632-10637 (2009).

79. Fabry, B. et al. Scaling the microrheology of living cells. Phys Rev Lett 87, 148102 (2001).

80. Johnston, R. A. et al. Allergic airway responses in obese mice. Am J Respir Crit Care Med 176, 650658 (2007).

81. Johnston, R. A., Zhu, M., Hernandez, C. B., Williams, E. S. \& Shore, S. A. Onset of obesity in carboxypeptidase E-deficient mice and effect on airway responsiveness and pulmonary responses to 


\section{Figures}

Fig 1
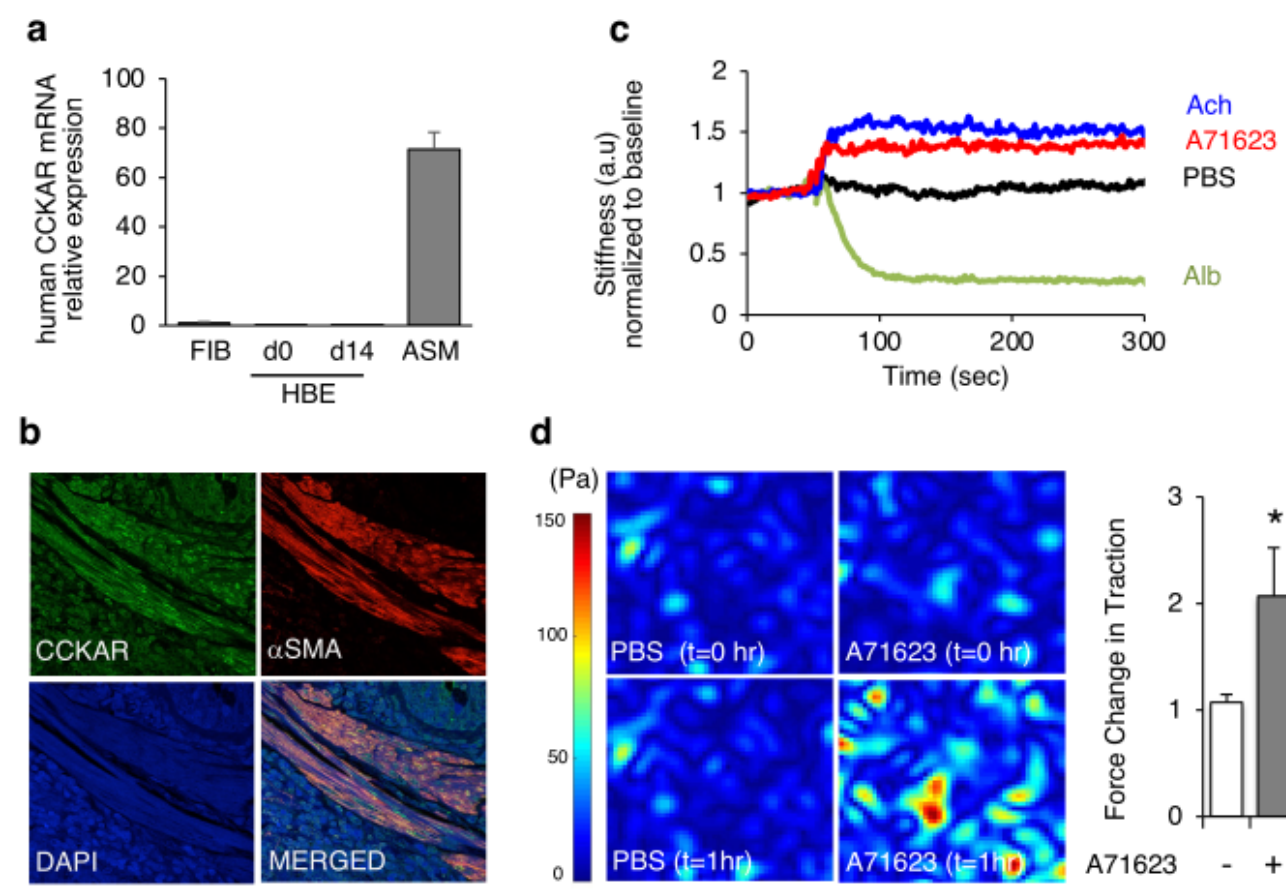

d

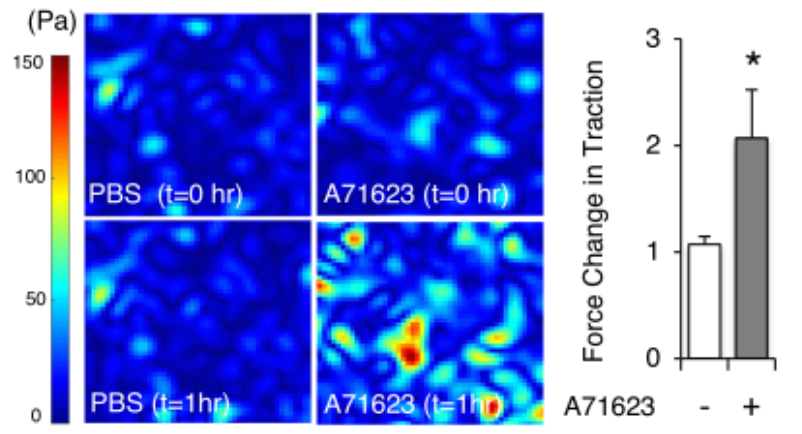

\section{Figure 1}

ASM cells express CCKAR and contract in response to CCK agonist. (a) qRT measurement of CCKAR mRNA expression in different airways cell types. FIB: primary lung fibroblasts; HBE: human bronchial epithelial cells [d0: day 0 of initiation of air-liquid interface (ALI) culture; d14: day 14 post-ALI]; ASM: airway smooth muscle cells. ( $n=3$ ) (b) Immunostaining of human lung tissue section for CCKAR (green). a-SMA (a-smooth muscle actin, red) was used as a marker of ASM cells. DAPI was used to stain the nuclei (blue). (c) OMTC-based measurement of cell stiffness in ASM cells treated with CCK agonist A71623 (10 nM), acetylcholine (Ach), $\beta$-agonist (Alb: albuterol) or PBS (vehicle). a.u.: arbitrary unit. (d) Representative TFM images in ASM cells after treatment with A71623 (10 nM) or PBS. Graph to the right is the quantification of TFM-based contractility measurement in control and A71623-treated ASM cells. * $p$ $<0.05$. 
Fig 2

a

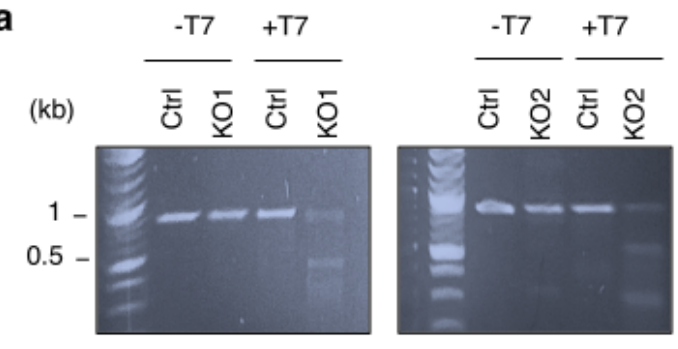

b
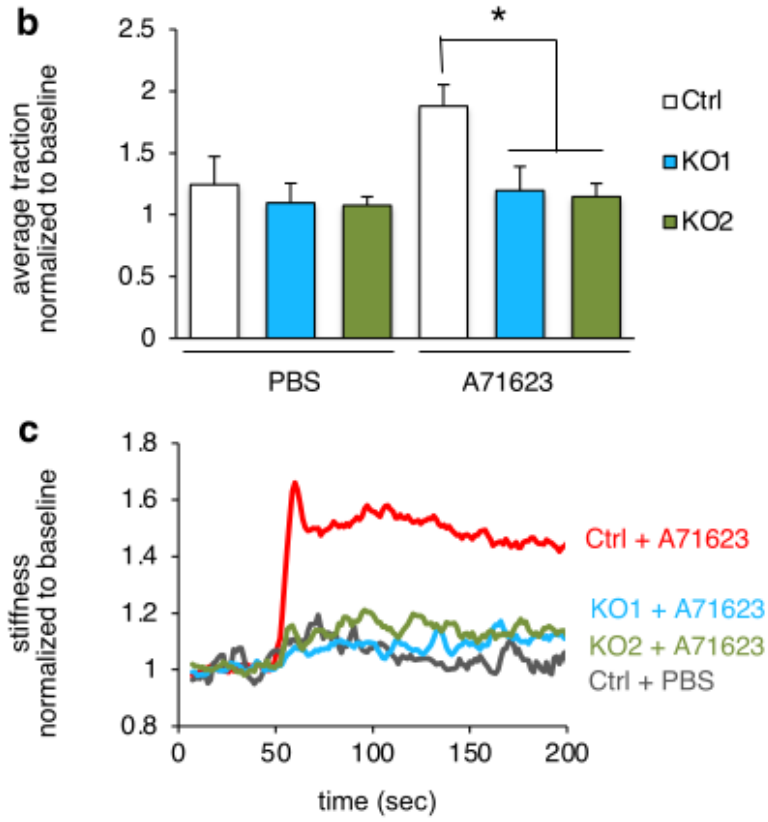
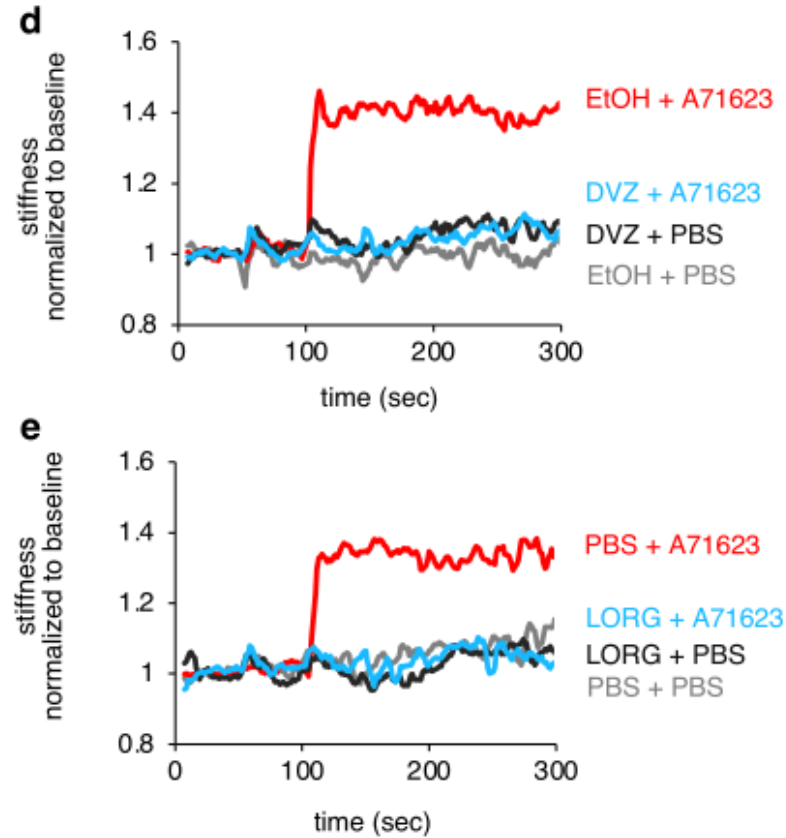

\section{Figure 2}

CCKAR mediates CCK-induced stiffening and contraction of ASM cells. (a) T7E1 assay on CRISPR/Cas9mediated CCKAR knockout ASM cells. CCKAR knockout (KO) cells were generated by lentiviral delivery of gRNAs targeting the CCKAR gene. DNA cleavage indicating mismatch and mutation induced by the two gRNAs targeting CCKAR was detected by T7 Endonuclease 1. Multiple bands in the DNA gel indicate mutation in the CCKAR gene in KO cells (KO1 and KO2). (b) TFM-based contractility measurement in control vs. CCKAR-KO ASM cells in response to A71623 treatment $(10 \mathrm{nM})$. $(n=4) * p<0.05$. (c) OMTCbased measurement of cell stiffness in control vs. CCKAR-KO cells in response to A71623 treatment (100 $n M)$. (d-e) OMTC-based measurement of cell stiffness in response to A71623 treatment (100 nM) and in the presence of devazepide (d) or lorglumide (e). Ethanol and PBS were used as vehicles for devazepide and lorglumide, respectively. dvz: devazepide, EtOH: ethanol, a.u.: arbitrary unit. 
Fig 3
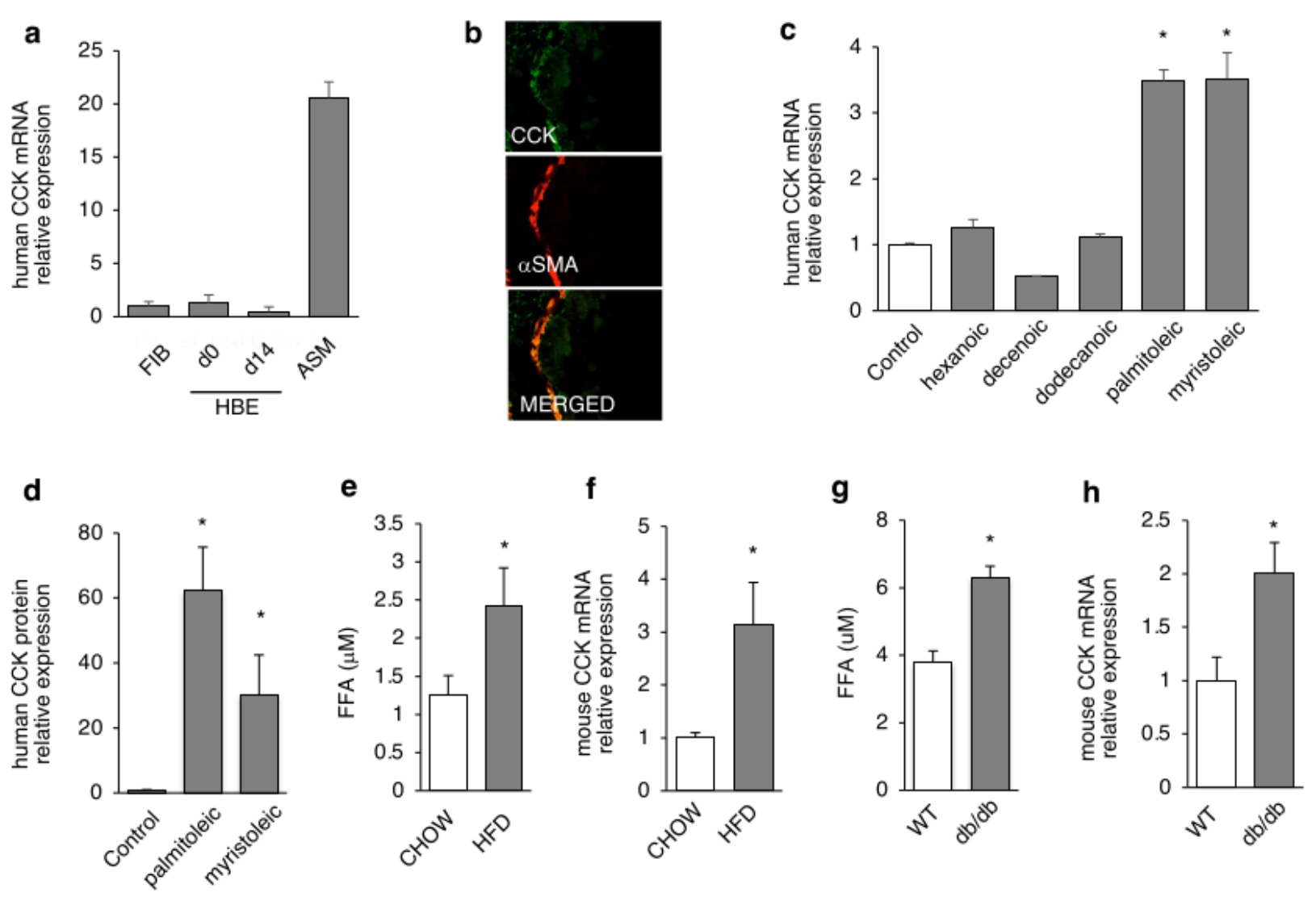

\section{Figure 3}

(a) qRT measurement of CCK mRNA expression in different airways cell types. FIB: primary lung fibroblasts; HBE: human bronchial epithelia cellsl; [d0: day 0 of initiation of air-liquid interface (ALI) culture; d14: day 14 post-ALI]; ASM: airway smooth muscle cells. $(n=3)(b)$ Immunostaining of mouse lung tissue section for CCK (green). a-SMA (a-smooth muscle actin, red) was used as a marker of ASM cells. (c) qRT measurement of CCK mRNA expression in ASM cells after treatment with indicated free fatty acids $(n=4) * p<0.05$. (d) ELISA measurement of CCK protein in medium of ASM cells after treatment with indicated free fatty acids $(n=4) * p<0.05$ (e) Quantification of free fatty acid (FFA) in bronchioalveolar lavage (BAL) fluid of regular chow-fed mice $(n=9)$ vs. HFD-fed mice $(n=13) * p<0.05(f)$ qRT measurement of CCK mRNA expression in in the lungs of regular chow-fed mice $(n=6)$ vs. HFD-fed mice $(n=6) * p<0.05$ (g) Quantification of free fatty acid (FFA) in bronchioalveolar lavage (BAL) fluid of wild type control mice $(n=4)$ vs. $d b / d b$ mice $(n=4)$ compared to wild type controls $(n=4) * p<0.05(h) q R T$ measurement of CCK mRNA expression in in the airways (lungs and trachea) of wild type control mice $(n=4)$ vs. $d b / d b$ mice $(n=4)$ compared to wild type control $(n=4) * p<0.05$. Actin or 18 s rRNA was used as reference gene. Results represent the mean \pm SEM. 
Fig 4

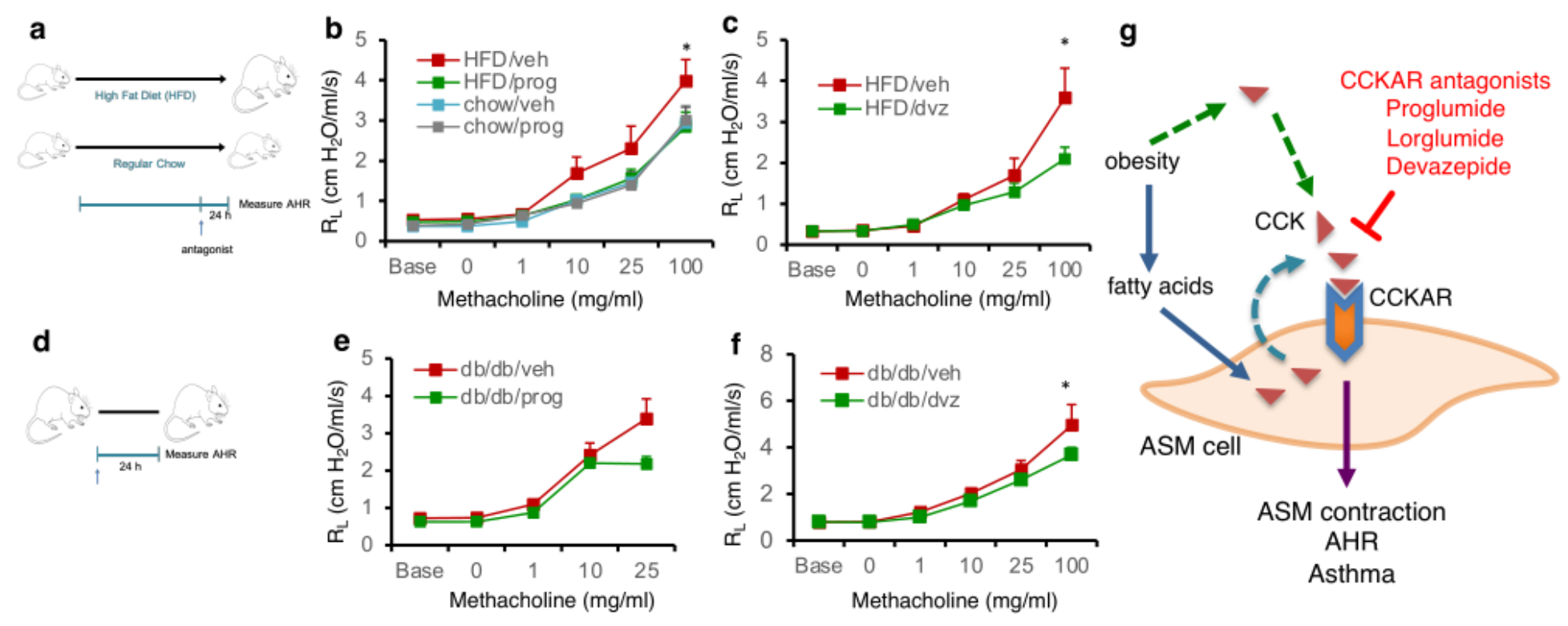

\section{Figure 4}

CCK contributes to innate AHR in mouse models of obesity-associated AHR. (a) Schematic diagram showing induction of obesity in mice and treatment with proglumide before Flexivent measurement. 8week old C57BL/6J mice were switched to high fat diet for at least 13 weeks. Mice were then given proglumide ( $50 \mathrm{mg} / \mathrm{kg}$, intranasal, $3 \mathrm{x}$ ) or devazepide $(25 \mu \mathrm{g} / \mathrm{kg}$, intranasal, $2 \mathrm{x}$ ) at a total volume of $25 \mu \mathrm{l}$ (12.5 $\mu \mathrm{l}$ in each nostril). Two hours after the last treatment, Flexivent was performed to assess AHR in response to increasing doses of methacholine (b). Pulmonary resistance of HFD-fed or regular chow-fed mice treated with proglumide $(n=11)$ or vehicle $(n=12)$. Results represent the mean \pm SEM. ${ }^{*} p<0.05$. (c) Pulmonary resistance of HFD-fed mice treated with devazepide $(n=9)$ or vehicle $(n=9)$. Results represent the mean \pm SEM. ${ }^{*} p<0.05$. (d) Scheme of treatment with proglumide or devazepide in $\mathrm{db} / \mathrm{db}$ mice or wild type control mice. 10-12 week old db/db mice were given proglumide $(50 \mathrm{mg} / \mathrm{kg}$, intranasal, $3 \mathrm{x}$ ) or devazepide $(25 \mu \mathrm{g} / \mathrm{kg}$, intranasal, $2 \mathrm{x})$. Two hours after the last treatment, Flexivent was performed to assess AHR in response to increasing doses of methacholine (e) Pulmonary resistance of $\mathrm{db} / \mathrm{db}$ mice treated with proglumide $(n=8)$ or vehicle $(n=9)$. Results represent the mean \pm SEM. ${ }^{*} p<0.05$ (f) (e) Pulmonary resistance of $\mathrm{db} / \mathrm{db}$ mice treated with devazepide $(\mathrm{n}=11)$ or vehicle $(\mathrm{n}=12)$. Results represent 
the mean \pm SEM. ${ }^{*} p<0.05$ (g) Possible mechanism of obesity-associated AHR. Obesity may induce CCK in ASM cells through circulating free fatty acids (FFA) or independent of FFA. This leads to secretion and binding of CCK to CCKAR expressed on the surface of ASM cells which in turn results in contraction of ASM, the main effector cells of bronchoconstriction and AHR. Antagonizing CCKAR (e.g. by pharmacological inhibitors) blocks CCK-induced ASM contraction and reduces AHR in the obese.

\section{Supplementary Files}

This is a list of supplementary files associated with this preprint. Click to download.

- SupplementaryTable.docx

- CCKSupplementaryFiguresandLegends.pdf 